\title{
A FACTORIZATION THEOREM FOR HOLOMORPHIC FUNCTIONS OF POLYNOMIAL GROWTH IN A HALF PLANE
}

\author{
BY E. J. BELTRAMI AND M. R. WOHLERS
}

Communicated by W. Rudin, April 12, 1965

A function $F(p), p=\sigma+i \omega$, belongs to class $H^{+}$, if it is holomorphic in the half plane $\operatorname{Re} p>0$, and if it is bounded by a polynomial uniformly in every half plane $\operatorname{Re} p \geqq \sigma>0$. The significance of the $H^{+}$ class is due to an important theorem of L. Schwartz [1]; this theorem tells us that $\mathrm{H}^{+}$characterizes the collection of Laplace transforms of certain distributions in $D_{+}^{\prime}$ (here, as in what follows, we use the terminology and notation of distributions to be found in [2]). Moreover, $H^{+}$is basic in the following extension of an $L_{2}$ result of PaleyWiener.

ThEOREM 1 [3]. A necessary and sufficient condition, in order that $F_{\omega} \in S^{\prime}$ (tempered distribution) be the boundary value in the $S^{\prime}$ topology of an $H^{+}$function $F(\sigma+i \omega)$, as $\sigma \rightarrow 0$, is that $F_{\omega}$ be the Fourier transform of some $\hat{F} \in S^{\prime} \cap D_{+}^{\prime}$. In particular, $F(p)$ is the Laplace transform of $\hat{F}$.

The classical version of Theorem 1 is obtained when $S^{\prime}$ is replaced by $L_{2} \subset S^{\prime}$ and $H^{+}$is replaced by the Hardy class $H^{2} C H^{+}$. Now a function which is of class $H^{2}$, in the right half plane, also admits a factorization into inner and outer factors, as given by a well-known theorem (cf. [4, p. 67]). The inner factor is a.e. of modulus one on the boundary and can be written as the product of a singular function and a Blaschke product taken with the zeros of the $H^{2}$ function; the outer factor is nonzero and is itself in $H^{2}$. Actually, $H^{2}$ is completely characterized by such a factorization.

The purpose of this note is to extend the classical result to the $\mathrm{H}^{+}$ class by proving the following theorem.

TheOREM 2. $F(p) \in H^{+}$can be factored as $F(p)=p^{k} B(p) S(p) g(p)$ (where $k$ is some nonnegative integer, $B(p)$ is a convergent Blaschke product formed with the zeros of $F(p), S(p)$ is singular, $g(p)$ is nonzero and in $H^{+}$, and $g(p)$ has a $D_{L_{2}}^{\prime}$ boundary value taken in the $S^{\prime}$ topology as $\sigma \rightarrow 0)$, if and only if $F(p)$ has $S^{\prime}$ boundary values as $\sigma \rightarrow 0$.

Proof. Let $F(p) \in H^{+}$tend to $F_{\omega} \in S^{\prime}$ as $\sigma \rightarrow 0$. Then, for sufficiently large $m, F(p)=p^{m} f(p)$ and $f(p) \rightarrow{ }^{\prime} f_{\omega} \in D_{L_{2}}^{\prime}$ (cf. [3]). Now let $f_{n}(\omega)$ be the regularizations of $f_{\omega}$; then the inverse Fourier transforms $\mathfrak{F}^{-1} f_{n} \equiv \hat{f}_{n} \in L_{2}(0, \infty)$, since $f_{n} \in L_{2}$. Moreover (Paley-Wiener), the 
functions $f_{n}(p)=\mathfrak{F}\left(e^{-\sigma t} \hat{f}_{n}\right)$ belong to $H^{2}$ in the half plane $\operatorname{Re} p>0$ and can be factored into inner and outer functions as $h_{n}(p) g_{n}(p)$. On the boundary the function $h_{n}$ (and hence $h_{n}^{-1}$ as well) are all holomorphic on some open $\Omega$ so that $\Omega^{\prime}$ is of measure zero. Since $h_{n}^{-1}$ are nonvanishing on $\Omega$ and $\left|h_{n}^{-1}\right|=1$ a.e., we extract a sequence (also denoted by subscript $n$ ) which converges uniformly on every compact subset of $\Omega$ and hence, as follows easily, $h_{n}^{-1}$ converge in the $S^{\prime}$ topology to some $h^{-1}$ which is holomorphic on $\Omega$. Therefore $D^{l}\left(h_{n}^{-1}-h^{-1}\right) \rightarrow S^{\prime} 0$ for each $l$ th order derivative. We now invoke the fact that $D^{l}\left(h_{n}^{-1}-h^{-1}\right)$ can be written as $D^{i}\left(1+\omega^{2}\right)^{N / 2} u_{n}(\omega)$ for some fixed $j, N$, and where $u_{n}$ converge uniformly to zero on the entire axis (cf. [5, p. 91] for a proof). Moreover, it is clear that the $u_{n}$ are actually holomorphic on $\Omega$. Hence we can estimate $D^{l}\left(h_{n}^{-1}-h^{-1}\right)$, using the Leibnitz rule, and obtain that $D^{l} h_{n}^{-1}=O\left(|\omega|^{M}\right)$ for some fixed $M$, since $D^{l} h^{-1}$ is itself tempered. Similarly, $f_{n}$ have the representation $D^{i}\left(1+\omega^{2}\right)^{N / 2} \tilde{f}_{n}$ for some other fixed $j, N$, where $\tilde{f}_{n}$ are now uniformly bounded on the axis. From these facts we find that if $\phi \in S$ (smooth functions of rapid decay at infinity), the integral of $g_{n}$ with $\phi$, on the boundary, is bounded in $n$, since $g_{n}=f_{n} h_{n}^{-1}$. Thus $\left\{g_{n}\right\}$ is bounded in the $S^{\prime}$ topology, and we can extract yet another sequence, again denoted by subscript $n$, which converges in the strong $S^{\prime}$ topology to some distribution $g$ (note that $S^{\prime}$ is a Montel space). Since $\hat{g}_{n} \in L_{2}(0, \infty)$, it follows that $\hat{g} \in S^{\prime} \cap D_{+}^{\prime}$. Moreover, $e^{-p t}$ is a bounded set in $S$ for $p \in K, K$ compact, and $t>0$; therefore, the Laplace transforms $g_{n}(p)$, or outer factors of $f_{n}(p)$, converge to $g_{0}(p)$, the transform of $\hat{g}$, uniformly in each compact subset of $\operatorname{Re} p>0$. Hence $g_{0}(p) \in H^{+}$, and, by Theorem $1, g_{0}(p)$ has an $S^{\prime}$ limit as $\sigma \rightarrow 0$. As indicated at the beginning of the proof, every such $g_{0}(p)$ can be written as $p^{r} g(p)$ for some nonnegative $r$, and where $g(p) \in H^{+}$has an $S^{\prime}$ boundary value in $D_{L_{2}}^{\prime}$. Now the Vitali-Montel theorem shows that the inner functions converge uniformly on each compact subset of the half plane to some holomorphic $h(p)$, so that $|h(p)| \leqq 1$. A theorem of Hurwitz [6, p. 119], then shows that $h(p)$ and $f(p)$ have identical zeros. Moreover, since $\left|h_{n}(\omega)\right|=1$ on $\Omega$, so is $|h(\omega)|=1$ a.e.; $h(p)$ is an inner function which can be written as the product of a convergent Blaschke $B(p)$ and a singular function $S(p)$. This establishes sufficiency, with $k=m+r$.

Conversely, if $F(p) \in H^{+}$has the given factorization, then for $h=B S, h(p) g(p)=\mathfrak{F}\left(e^{-\sigma t}(\hat{h} * \hat{g})\right)$, where $\hat{h}, \hat{g} \in D_{+}^{\prime}$. Since $h(\omega) / 1+i \omega$ $\in L_{2}$, it follows that $\hat{h} \in D_{L_{2}}^{\prime}$, while $\hat{g}$ is a locally $L_{2}$ tempered function, since $g \in D_{L_{2}}^{\prime}$. Thus $\hat{h} * \hat{g} \in S^{\prime} \cap D_{+}^{\prime}$ and, by Theorem $1, F(p)$ has an $S^{\prime}$ limit as $\sigma \rightarrow 0$, since $p^{k}$ trivially does. 
It is worthwhile noting that the logarithm of the outer factor $p^{r} g(p)$ exists, and is uniquely determined by the magnitudes of the $f_{n}$ on the boundary, since $\log \operatorname{pr}^{r} g(p)$ are uniquely determined by $\log \left|f_{n}(\omega)\right|$.

\title{
REFERENCES
}

1. L. Schwartz, Transformations de Laplace des distributions, Sem. Math. Univ. Lund, Tome Supp. (1952), 196-206.

2. —_ Theorie des distributions, Tome I, II, Hermann, Paris, 1957, 1959.

3. E. J. Beltrami and M. R. Wohlers, Distributional boundary value theorems and Hilbert transforms, Arch. Rational Mech. Anal. 18 (1965), 304-309.

4. K. Hoff man, Banach spaces of analytic functions, Prentice-Hall, Englewood Cliffs, N. J., 1962.

5. A. Friedman, Generalized functions and partial differential equations, PrenticeHall, Englewood Cliffs, N. J., 1963.

6. E. C. Titchmarsh, The theory of functions, 2nd ed., Oxford Univ. Press, Oxford, 1939.

Grumman Aircraft Engineering Company

\section{THREE THEOREMS ON MANIFOLDS WITH BOUNDED MEAN CURVATURE}

\author{
BY F. J. ALMGREN, JR.
}

Communicated by J. Moser, May 27, 1965

The following are three theorems about manifolds having bounded mean curvature which illustrate some of the applications to classical differential geometry of the structure theorems for regular integral varifolds. The proofs, which will appear in $[\mathbf{A}]$, are geometric and measure theoretic. Let $2 \leqq k \leqq n$ be integers.

THEOREM 1. There exist numbers $a(k)>0$ and $b(k, n)<\infty$ with the following property: Let $A$ be a compact k-dimensional manifold of class $C^{2}$ with boundary $B$ and $f: A \rightarrow R^{n}$ be a $C^{2}$ immersion of $A$ into $R^{n}$ having mean curvature no larger than $M$ at each point. If

$$
M^{k}[k \text {-area of } f \mid A] \leqq a(k),
$$

then

$$
[k \text {-area of } f \mid A] \leqq b(k, n)[(k-1) \text {-area of } f \mid B]^{k /(k-1)} .
$$

In particular, if $f$ satisfies the minimal surface equation, then, without additional hypotheses, 